\title{
Immucillin-H binding to purine nucleoside phosphorylase reduces dynamic solvent exchange
}

\author{
FANG WANG,${ }^{1,2}$ ROBERT W. MILES, ${ }^{2}$ GREGORY KICSKA, ${ }^{2}$ EDWARD NIEVES, ${ }^{1,2}$ \\ VERN L. SCHRAMM, ${ }^{2}$ AND RUTH HOGUE ANGELETTI ${ }^{1,2,3}$ \\ ${ }^{1}$ Laboratory for Macromolecular Analysis and Proteomics, Albert Einstein College of Medicine, Bronx, New York 10461 \\ ${ }^{2}$ Department of Biochemistry, Albert Einstein College of Medicine, Bronx, New York 10461 \\ ${ }^{3}$ Department of Developmental \& Molecular Biology, Albert Einstein College of Medicine, Bronx, New York 10461
}

(Received March 29, 2000; Final Revision May 30, 2000; AccePted June 15, 2000)

\begin{abstract}
The rate and extent of hydrogen/deuterium (H/D) exchange into purine nucleoside phosphorylase (PNP) was monitored by electrospray ionization mass spectrometry (ESI-MS) to probe protein conformational and dynamic changes induced by a substrate analogue, products, and a transition state analogue. The genetic deficiency of PNP in humans is associated with severe T-cell immunodeficiency, while B-cell immunity remains functional. Inhibitors of PNP have been proposed for treatment of T-cell leukemia, to suppress the graft-vs.-host response, or to counter type IV autoimmune diseases without destroying humoral immunity. Calf spleen PNP is a homotrimer of polypeptide chains with 284 amino residues, molecular weight 31,541. Immucillin-H inhibits PNP with a $K_{d}$ of 23 pM when only one of the three catalytic sites is occupied. Deuterium exchange occurs at 167 slow-exchange sites in $2 \mathrm{~h}$ when no catalytic site ligands are present. The substrate analogue and product prevented H/D exchange at 10 of the sites. Immucillin-H protected 32 protons from exchange at full saturation. When one of the three subunits of the homotrimer is filled with immucillin-H, and 27 protons are protected from exchange in all three subunits. Deuterium incorporation in peptides from residues 132-152 decreased in all complexes of PNP. The rate and/or extent of deuterium incorporation in peptides from residues 29-49, 50-70, 81-98, and 112-124 decreased only in the complex with the transition state analogue. The peptidespecific H/D exchange demonstrates that (1) the enzyme is most compact in the complex with immucillin- $\mathrm{H}$, and (2) filling a single catalytic site of the trimer reduces H/D exchange in the same peptides in adjacent subunits. The peptides most highly influenced by the inhibitor surround the catalytic site, providing evidence for reduced protein dynamic motion caused by the transition state analogue.
\end{abstract}

Keywords: H/D exchange; immucillin-H; mass spectrometry; negative cooperativity; one-third-the-sites reactivity; purine nucleoside phosphorylase

The genetic deficiency of purine nucleoside phosphorylase (PNP) results in the failure of T-cell development in humans (Hershfield \& Mitchell, 1995). The hypothesis for the phenotype is that accumulation of dGTP in immature T-cells prevents the balanced synthesis of deoxynucleotides by ribonucleotide reductase, and halts cell division of the T-cell precursors. PNP is the only enzyme known to degrade deoxyguanosine in humans, and T-cells are efficient at converting deoxyguanosine to dGTP. The genetic disease is remarkably specific for T-cells and is thought to be a conse-

Reprint requests to: Ruth Hogue Angeletti, Laboratory for Macromolecular Analysis and Proteomics, Albert Einstein College of Medicine, 1300 Morris Park Avenue, Bronx, New York 10461; e-mail: angelett@ aecom.yu.edu.

Abbreviations: H/D, hydrogen/deuterium; HPLC, high-performance liquid chromatography; MS, mass spectrometry; LC-MS, liquid chromatography coupled to mass spectrometry; MS/MS, tandem mass spectrometry; PNP, purine nucleoside phosphorylase. quence of deoxynucleoside kinase(s), which phosphorylate deoxyguanosine, and the lack of a dGMP phosphatase, which has been reported in other tissues. B-Cells appear normal, and erythrocytes have normal function, even though dGTP is also elevated in erythrocytes.

The search for efficient PNP inhibitors is driven by the proposal that T-cell responses might be weakened with less effect on other tissues (Morris \& Montgomery, 1998). Type IV autoimmune disorders, T-cell lymphoma, and tissue transplant rejection are possible uses for such inhibitors. Recent application of transition state theory to the PNP reaction has led to the development of the immucillins, transition state inhibitors for mammalian PNPs that bind with picomolar affinity (Miles et al., 1998). Complete inhibition of trimeric PNP occurs with a single subunit filled with immucillin- $\mathrm{H}$, supporting a sequential activity at the trimer subunits (Miles et al., 1998). In contrast, X-ray crystal structures of substrate, product, and inhibitor complexes reveal saturation at all three catalytic sites (Koellner et al., 1997; Mao et al., 1998). This 
work tests the hypothesis that transition state analogue inhibitors differ from substrate or product analogues by causing conformational changes that are characteristic of the transition state. The experimental approach is to determine the extent to which the peptide bonds exchange protons in the presence of a substrate analogue, the products of the reaction, and a transition-state analogue inhibitor. Exchange rates are measured at partial and full catalytic site saturation for the transition-state analogue.

Transition-state theory for enzyme-catalyzed reactions proposes that the distorted substrate is bound with high affinity at the transition state. It is further proposed that this binding energy is accompanied by a mutual distortion of the enzyme and substrate to achieve the transition-state complex. Transition-state affinity is captured in analogues that are similar to the transition state, but with a chemically stable structure. It is now possible to test these hypotheses for PNP, because the immucillin transitionstate analogue inhibitors bind approximately one million times tighter to the enzyme than substrate and exhibit slow-onset inhibition and slow release, characteristic of transition state analogue interactions (Miles et al., 1998; Morrison \& Walsh, 1988). The reaction product hypoxanthine, and the substrate analogue formycin B can be used to compare the effects of Michaelis complexes with the transition-state analogue complex formed with immucillin-H (Fig. 1).

Hydrogen/deuterium (H/D) exchange of proteins coupled with mass spectrometry has been used to study the dynamics of protein folding of model proteins (Katta \& Chait, 1993; Zhang \& Smith, 1993; Zhang et al., 1996; Smith et al., 1997), and more recently, to identify molecular details of protein function (Wang et al., 1997, 1998a, 1998b, 1999; Mandell et al., 1998a, 1998b; Woodward, 1999). Peptide contacts in substrate and inhibitor binding sites have been identified as have conformational changes in "hinge" regions of enzymes (Wang et al., 1997, 1998b). Although H/D exchange analysis by mass spectrometry is not a high-resolution technology, it provides dynamic structural information that is difficult to obtain by other methods. Region-specific information is obtained by examination of specific peptides. In the present investigation, $\mathrm{H} / \mathrm{D}$ exchange has been used to identify a more protected conformational state in PNP when incubated with a high affinity transition-state analogue. The analogue exhibits one-third-the-sites inhibition of PNP, and H/D exchange is used to establish that global conformational changes occur as a consequence of binding a single inhibitor molecule per trimer.
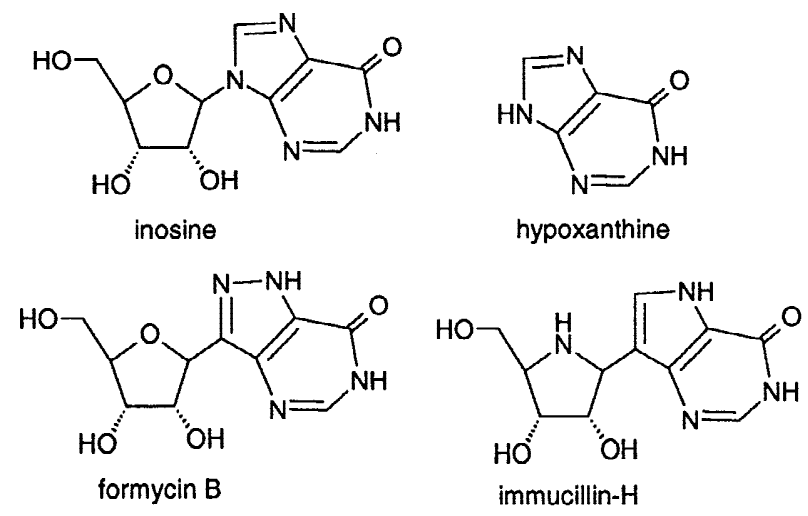

Fig. 1. Structure of ligands used in PNP complex formation.
Results

\section{Global $H / D$ exchange properties of PNP}

The global H/D exchange for PNP was analyzed in the absence and presence of the substrate, transition state analogues, and products. Fully deuterated PNP was used to normalize the exchange results. Deutero-PNP was obtained by heating in the presence of $2 \mathrm{M}$ urea- $\mathrm{d}_{4}$ in 95 atom\% excess $\mathrm{D}_{2} \mathrm{O}$ for $1.5 \mathrm{~h}$ at $55^{\circ} \mathrm{C}$. This sample was used to determine the time-dependent deuterium loss during the experimental steps of quenching, pepsin digestion, and liquid chromatography-mass spectrometry (LCMS) of deuterated PNP. The extent of H/D exchange into intact PNP is compared to the complexes of PNP with formycin B, hypoxanthine, and immucillin-H (Fig. 2). Complexes of PNP with near-saturating substrate analogue or product showed similar decreases in deuterium incorporation compared to unliganded PNP. Substrate and product complexes decreased by 10 the amount of deuterium incorporated into the PNP complexes at $2 \mathrm{~h}$ of exchange period. Deuterium incorporation into the complex of PNP with excess immucillin-H was decreased at all time points relative to the other conditions. In the PNP-immucillin-H complex, $\sim 22$ exchangeable amide hydrogens of each polypeptide chain are protected at $2 \mathrm{~h}$ against solvent exchange relative to complexes with substrate or product.

\section{Identification of peptides with differences in deuterium incorporation}

Solvent exchange, followed by proteolysis at low $\mathrm{pH}$, provides information on solvent accessibility of specific peptides. Pepsin digestion, followed by LC-MS analysis at reduced temperature, was employed to identify the regions of the PNP polypeptide that are protected from $\mathrm{H} / \mathrm{D}$ exchange by these compounds.

Peptic digests following solvent exchange were analyzed for peptides whose masses were changed in the complexes with respect to the free enzyme. Time points from $3 \mathrm{~min}$ of $\mathrm{H} / \mathrm{D}$ exchange to $210 \mathrm{~min}$ were analyzed. Five peptides with altered masses were resolved. For each of these masses, several peptides within the amino acid sequence could be assigned. The sequences were

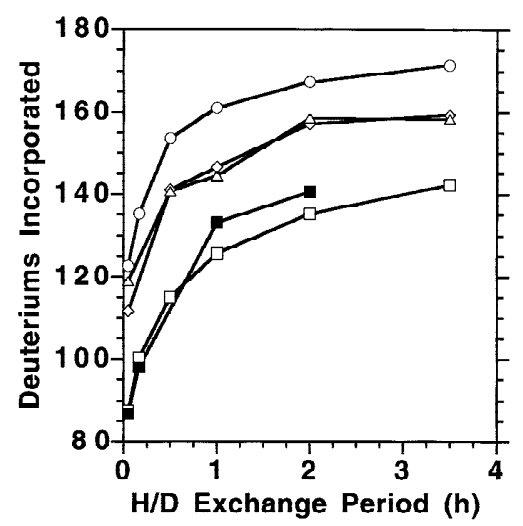

Fig. 2. The amount of deuterium incorporated at amide positions of intact enzyme as a function of time in $\mathrm{D}_{2} \mathrm{O}$. Exchange was accomplished for PNP (open circles), PNP in the presence of near-saturating formycin B (open triangles), hypoxanthine (open diamonds), or immucillin-H (open squares), or immucillin-H one-third the site inhibition (filled squares). 
identified by tandem mass spectrometry (MS/MS) analysis. One such analysis is demonstrated in Figure 3. In Figure 3A, peptide sequences whose masses fall within experimental error of the observed mass of 2,326 are identified. The MS/MS spectrum of HPLC-purified peptide obtained from the pepsin digestion of PNP is shown in Figure 3B and identified as corresponding to residues 81-98. Figure 4 shows the position in the amino acid sequence of the five peptides that were determined by MS/MS analysis to demonstrate differences in mass between the free and liganded PNP.

\section{Peptide with altered $H / D$ exchange in all complexes}

The peptide corresponding to the amino acid sequence 132-152 showed altered deuterium incorporation when PNP is incubated in the presence of substrate analogue, product, or transition state analogue (Fig. 5). At $2 \mathrm{~h}$ of exchange, compared to the free enzyme, the number of deuteriums incorporated decreased by four in all three complexes, after correction for back exchange during the quenching, pepsin digestion, and LC-MS steps. The exchange re- sult establishes that this peptide has decreased access to solvent when the active site is occupied.

\section{Peptides with $H / D$ incorporation altered} only by immucillin- $H$

Peptides corresponding to the amino acid sequences $29-49,50$ 70, 81-98, and 112-124 demonstrated reduced deuterium incorporation only when PNP was complexed with immucillin-H. Deuterium exchange was essentially the same in the complexes of the substrate analogue and product (Fig. 6). Two peptides corresponding to residues $50-70$ and $81-98$ show the most dramatic changes in $\mathrm{H} / \mathrm{D}$ exchange. These differences were best quantitated after the deuterium uptake vs. time profile was subjected to maximum entropy method analysis to show the relative distribution of the rate constants of exchangeable amide hydrogens (Zhang et al., 1997) (Fig. 7). This mode of representation indicates the kinetics of the exchange reactions. The ordinate in Figure 7 is scaled so that the total area under each peak corresponds to the total number of amide hydrogens over the range of exchange rate constants span-

\begin{tabular}{cccl}
\hline Molecular weight & \multicolumn{1}{c}{ Fragment } & \multicolumn{1}{c}{ Sequence } \\
2325.7 & {$[\mathrm{D}] 13-33[\mathrm{G}]$} & TAKWLLSHTEQRPQVAVICGS \\
2326.6 & {$[\mathrm{G}] 38-57[\mathrm{E}]$} & LVNKLTQAQTFDYSEIPNFP \\
2325.6 & {$[\mathrm{Y}] 51-72[\mathrm{~L}]$} & SEIPNFPESTVPGHAGRLVFGI \\
2325.7 & {$[\mathrm{M}] 81-98[\mathrm{P}]$} & MQGRFHMYEGYPFWKVTF \\
2325.6 & {$[\mathrm{~S}] 164-182[\mathrm{E}]$} & DAYDRDMRQKAHSTWKQMG
\end{tabular}

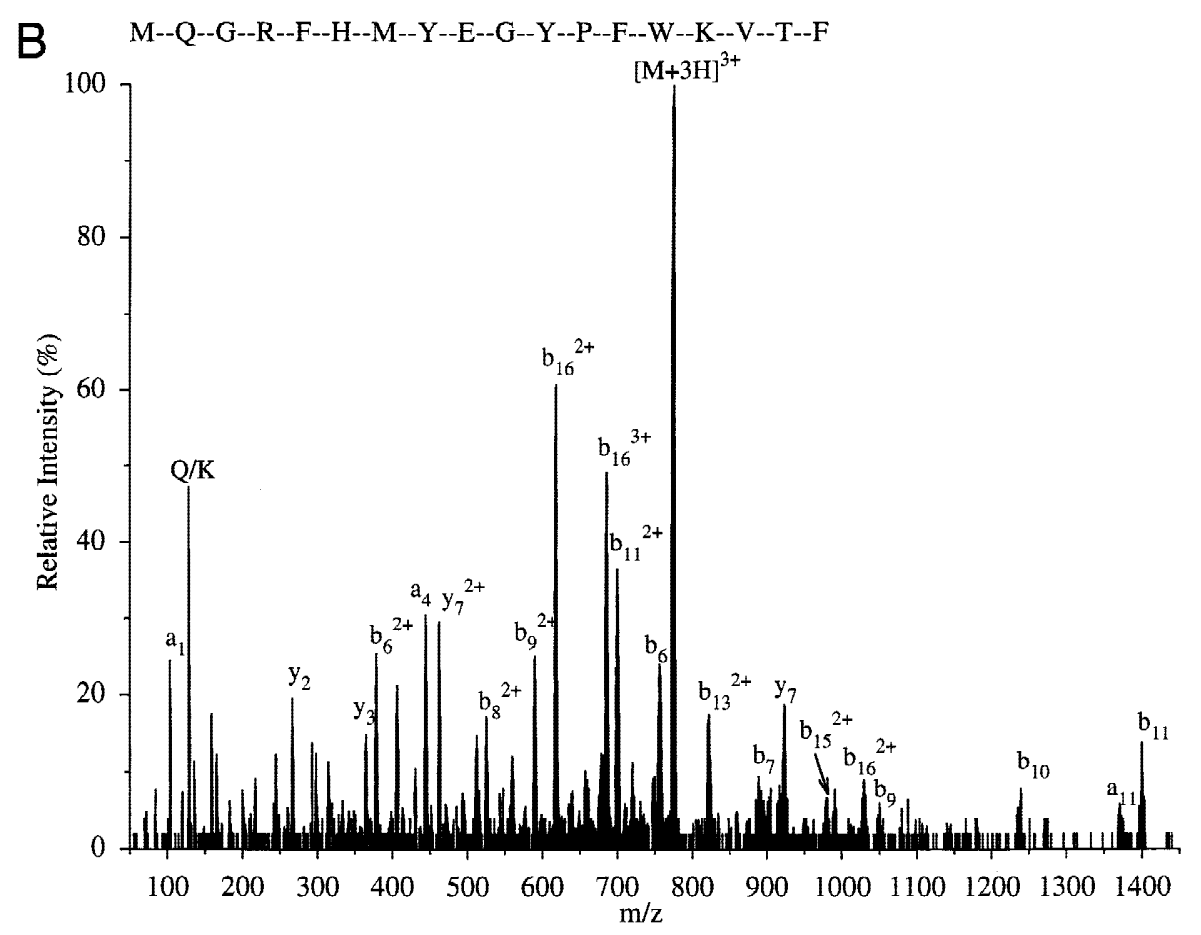

Fig. 3. An example of peptide identification. A: The candidate peptide sequences. B: The MS/MS spectrum of the triply protonated peptide representing residues 81-98 of PNP. Some of the fragment ions have been labeled according to the nomenclature proposed by Biemann (1988). 


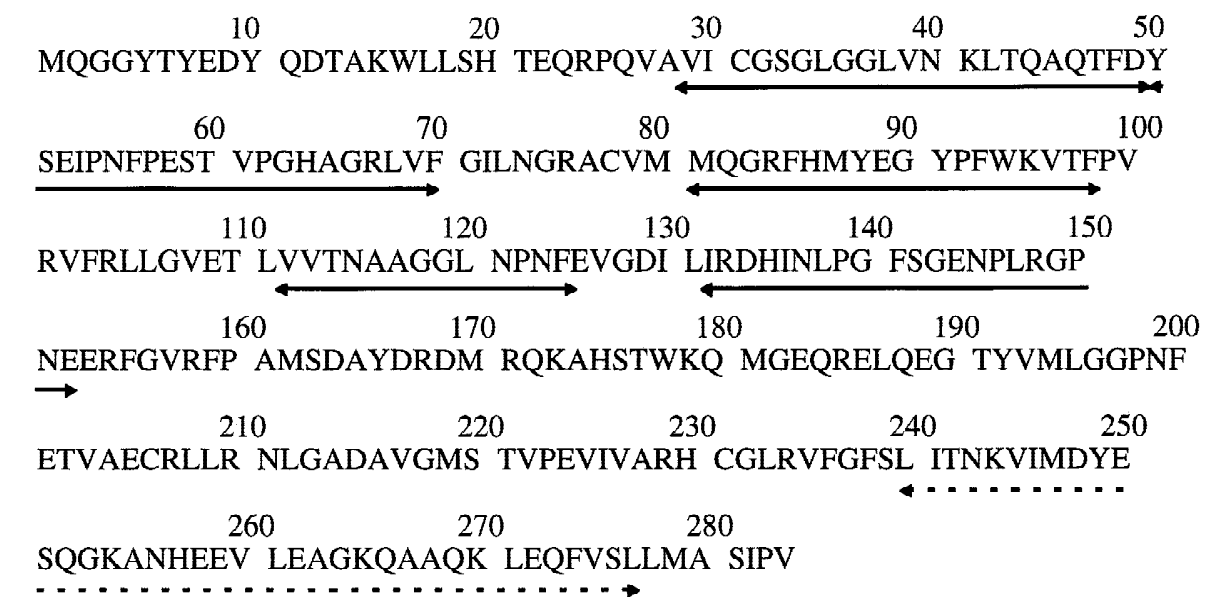

Fig. 4. Amino acid sequence of PNP indicating the position of peptides whose hydrogen/deuterium exchange was altered in complexes. The dashed line indicates a peptide of 4,260.8 $\mathrm{Da}$ identified among the acid insoluble peptic peptides.

ning that peak. For rate constants that are too slow (or too fast) to be determined from the experimental data, the rate constant distribution simply levels off to a horizontal line extending infinitely to the left (or right). The number of exchangeable hydrogens for each distinguishable rate constant is indicated above the lines in the graphs. For reference, the total number of potentially exchangeable amide hydrogens is listed in each panel for a given segment. For example, for peptide 50-70, four fast exchangeable protons in the free PNP and the Michaelis complexes slow down into two intermediately exchangeable protons and two slow exchangeable protons in the complex with transition state analogue.

\section{Stoichiometry of immucillin- $H$ binding}

Direct binding studies of $\left[{ }^{14} \mathrm{C}\right]$-immucillin-H reveal distinct affinity in binding to PNP. The first $K_{d}$ of $23 \mathrm{pM}$ titrates stoichiometrically as the first equivalent is added (Miles et al., 1998). The

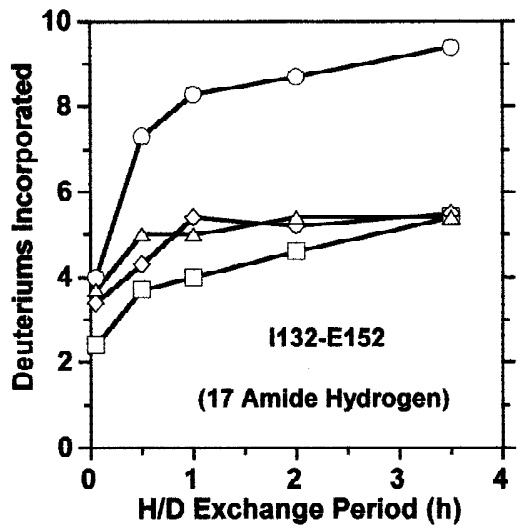

Fig. 5. The amount of deuterium incorporated at amide positions of peptic peptide I132-E152 as a function of time. No added compound (circle) formycin B (triangles); hypoxanthine (diamonds); immucillin-H (squares). second site has an apparent $K_{d}$ of $<5 \mu \mathrm{M}$ while the apparent $K_{d}$ of the third site is near $100 \mu \mathrm{M}$ (data not shown). Therefore, PNP demonstrates dramatic negative cooperativity as all three sites are filled with immucillin-H.

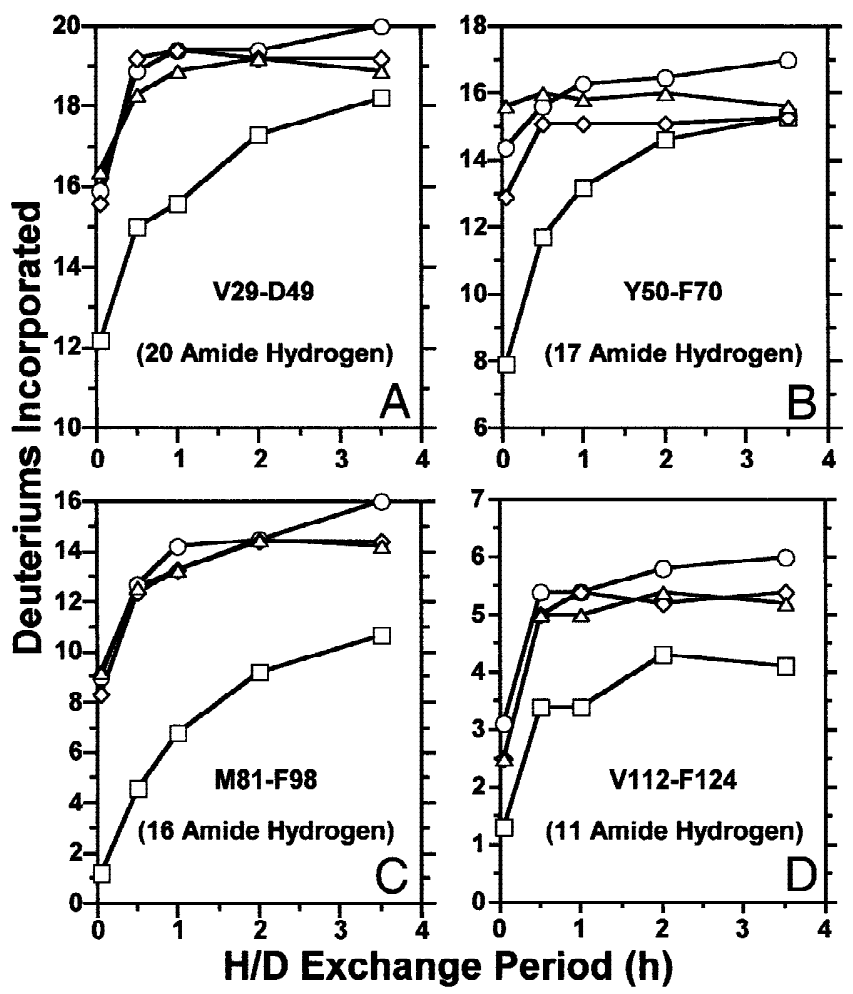

Fig. 6. The amount of deuterium incorporated at peptide amide positions as a function of time in peptides (A) V29-D49, (B) Y50-F70, (C) M81F98, and (D) V112-F124 . No added compound (open circle); formycin B (triangles); hypoxanthine (diamonds); immucillin-H (squares). 

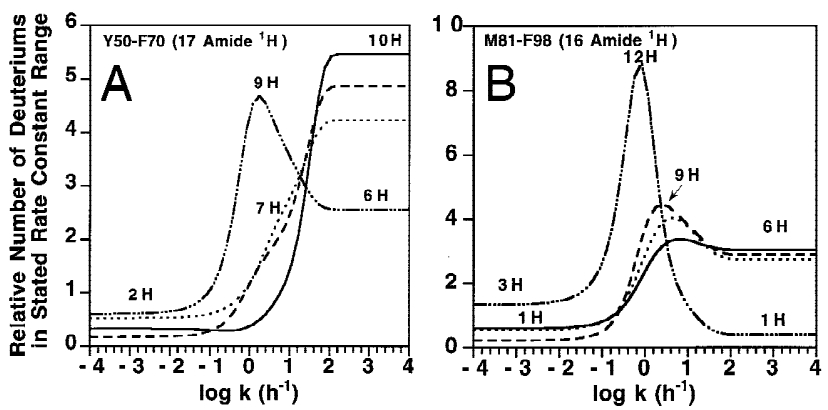

Fig. 7. H/D exchange rate constant distribution derived from the H/D exchange time courses shown in Figure 6, for peptides (A) Y50-F70 and (B) M81-F98. No added compound (- - ); formycin B (- - ; hypoxanthine (…..); immucillin-H $(-\cdots-)$. The total number of backbone amide hydrogens for each segment is indicated in each panel. The number of backbone amide hydrogens for each resolved peak in the rate constant distribution is shown above that peak.

\section{Comparison of global $H / D$ exchange in one-third} the sites and full saturation by immucillin- $H$

Negative cooperativity of inhibitor binding demonstrates restricted binding access to sites two and three as a consequence of immucillin-H binding at the first site. The phenomenon suggests restricted peptide motion that accompanies binding of the first ligand. Exchange experiments described above were initiated with $400 \mu \mathrm{M}$ PNP subunits and $800 \mu \mathrm{M}$ immucillin- $\mathrm{H}$, where all catalytic sites would be near saturation. Global exchange experiments with $400 \mu \mathrm{M}$ PNP subunits and $133 \mu \mathrm{M}$ immucillin-H fill only the first, tightly bound site $\left(K_{d}=23 \mathrm{pM}\right)$. The extent of deuterium incorporation was similar to the complex fully saturated with immucillin-H in the complex of onethird catalytic sites filled with immucillin-H (Fig. 2). Only a single molecular species was observed by mass spectrometry at each time point.

\section{Discussion}

Purine nucleoside phosphorylase is a homotrimer of polypeptide chains with 284 amino acid residues, molecular weight 31,541 . It has 269 exchangeable amide hydrogens. Hydrogen/deuterium exchange into intact PNP subunits indicates that 10 amide protons are protected from solvent exchange by hypoxanthine and formycin $\mathrm{B}$ (Fig. 2). Both of these substrate analogues provide equivalent effects. Immucillin-H is isosteric with formycin B (Fig. 1), but is a transition-state analogue inhibitor binding $\sim 10^{6}$ more tightly (Miles et al., 1998). The $K_{d}$ for the first binding site is $23 \mathrm{pM}$. The second and third sites have a distinctly weaker affinity, demonstrating negative cooperativity as all three sites are filled. Unlike substrate analogues or product, the transition-state analogue inhibitor is expected to induce conformational changes representing the $k_{\text {cat }}$ complex rather than the Michaelis complexes. Differences in H/D exchange will reflect the disposition of the peptides in Michaelis and catalytic conformation. Saturation with the transition-state analogue inhibitor protects 32 amide protons from solvent exchange (2 h) while substrate analogues protect 10 amide protons (Fig. 2). Binding of immucillin-H restricts motion in the protein structure, preventing solvent access to triple the sites protected by substrate analogues. Hydrogen/deuterium exchange into the five peptides provides specific information about these regions of the catalytic site and its response to formation of Michaelis complex compared to the transition state analogue complex. The peptides are indicated in the structure with bound inosine (Fig. 8), and their contacts in the enlargement of the active site region (Fig. 9). The involvement of each peptide in these complexes is described below.

\section{Peptide V29-D49}

Deuterium exchange in the peptide is rapid and exchange of all 20 amide protons is complete in $3.5 \mathrm{~h}$ (Fig. 6A). Addition of hypoxanthine or formycin B has no significant influence on the kinetics of exchange, except for the final proton. Thus, the access of solvent to this peptide is essentially unchanged in Michaelis complexes. Ser33 of the peptide forms two hydrogen bonds to phosphate (sulfate in the complex used here) (Fig. 9). However, these do not influence solvent access to the peptide. With bound immucillin- $\mathrm{H}$, $\mathrm{H} / \mathrm{D}$ exchange rate decreases, and two amide protons are prevented from exchange for a period of $2 \mathrm{~h}$, clearly implicating this peptide in the protein conformation, which converts the Michaelis complex to the transition state analogue complex.

\section{Peptide Y50-F70}

The kinetics of deuterium exchange are closely related to those of V29-D49 (Fig. 6B). The contact to the catalytic site is a single H-bond to phosphate from His64 (Fig. 9). Substrate analogue has no effect on this solvent-accessible and rapidly exchanging peptide, except for one final proton. Immucillin-H protects half of the exchangeable amide protons at early times and slows the exchange rate considerably. After binding of immucillin- $\mathrm{H}$, four fast exchangeable amide protons slow down to two intermediately exchangeable protons or two slow exchangeable protons (Fig. 7A). This pattern indicates a change in dynamic motion (rate effect) specific for the transition state analogue complex, and not for the Michaelis-like complex.

\section{Peptide M81-F98}

In the absence of catalytic site ligands, all 16 of the amide protons in this peptide exchange with solvent in $3.5 \mathrm{~h}$ (Fig. 6C), indicating full solvent access. Michaelis complexes with hypoxanthine or formycin B have no effect on the kinetics of exchange for the first 14 amide protons (over the first $2 \mathrm{~h}$ ), but ultimately protect two of the amide proton exchange sites from exchange at $3.5 \mathrm{~h}$. The protection of only two protons occurs despite contacts from both Arg84 and His86 to the phosphate oxygens and Tyr88 to the O3" of inosine (Mao et al., 1998) (Fig. 9). The results establish that the peptide maintains its solvent access for all but two protons in the Michaelis complex. With bound immucillin-H, eight of the amide protons that exchange after $3 \mathrm{~min}$ are protected. Following $2 \mathrm{~h}$ of exchange, deuterium incorporation is six fewer than in the Michaelis complexes at comparable times. Formation of the tightly bound inhibitor causes a marked kinetic shielding from solvent access. Five fast exchangeable hydrogens slow down into three intermediately exchangeable protons and two slow exchangeable protons (Fig. 7B). The solvent accessibility of the peptide is weakly influenced in the Michaelis complex, but strongly affected in the transition state analogue complex. The reaction coordinate for PNP involves translocation of $\mathrm{C}^{\prime}$ of the ribooxocarbenium formed at the transition state toward the phosphorus nucleophile. Peptide M81-F98 contacts both the phosphate and the ribosyl group, and 


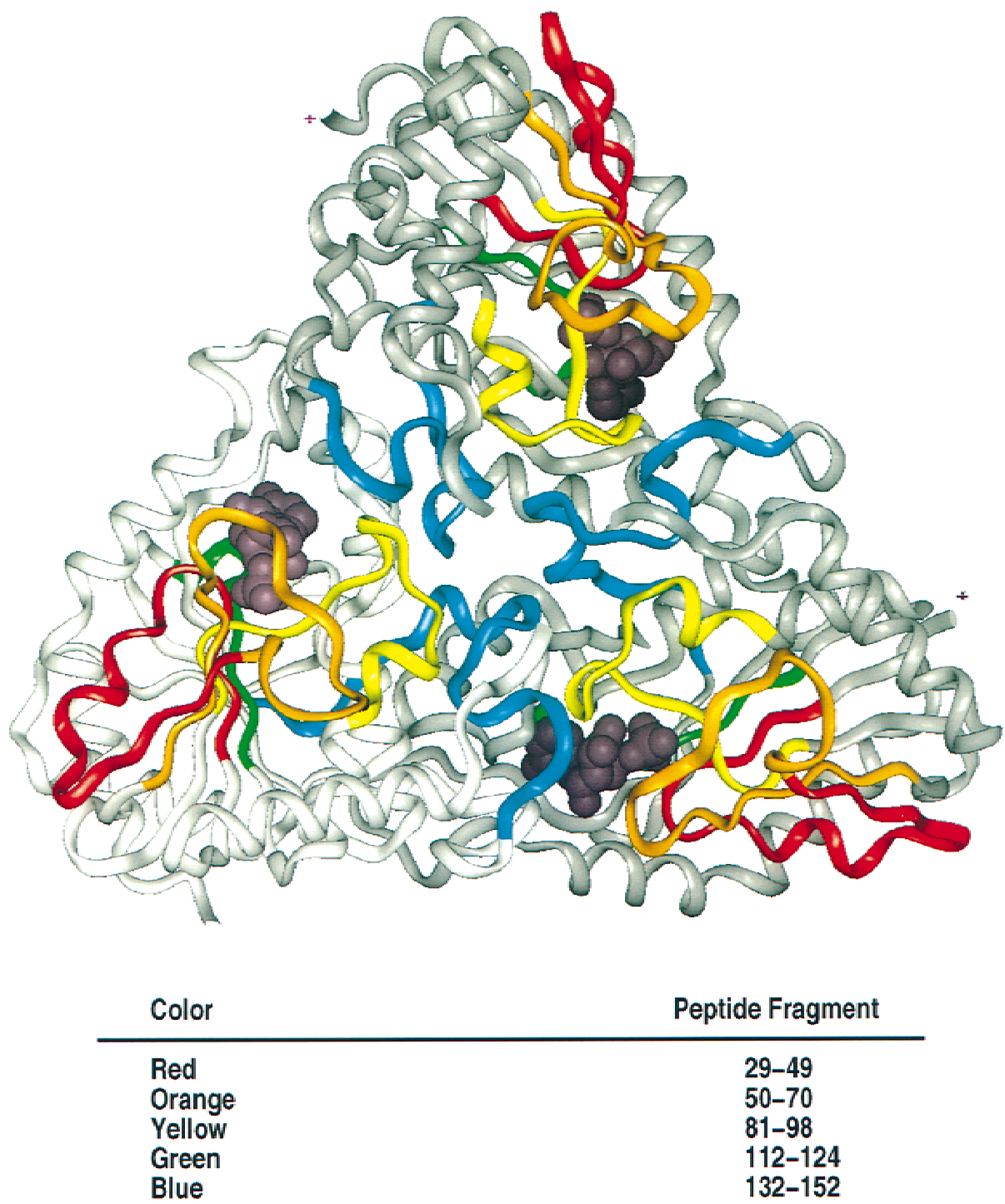

Fig. 8. Ribbon diagram of the three-dimensional structure of the PNP trimer in the presence of inosine and sulfate. All three subunits are shown; one polypeptide chain is distinguished by its lighter color. Space filling representations of inosine and sulfate are shown in purple. Peptide segments are colored as follows: red, 29-49; orange, 50-70; yellow, 81-98; green, 112-124; blue, $132-152$.

is the most strongly shielded against proton exchange by the transition state analogue inhibitor.

\section{Peptide V112-F124}

Five of the 11 exchangeable amide protons are unchanged after $3.5 \mathrm{~h}$ in unliganded PNP (Fig. 6D), consistent with a partially inaccessible peptide or a peptide having slow dynamics. The Michaelis complexes have no significant influence on the kinetics or extent of exchange. At $2 \mathrm{~h}$, immucillin- $\mathrm{H}$ protects two additional amide protons from exchange, indicating involvement in the structure of the transition state analogue complex to the extent of removing two peptide bonds from solvent access. The change in solvent access is likely to be related to interaction of the peptide nitrogen of Ala116 with one of the phosphate oxygens (Mao et al., 1998) (Fig. 9). The significant protection in the presence of the immucillin-H but not substrate interactions indicates that the mo- bility of this peptide is specifically decreased as a result of binding of the transition state analogue.

\section{Peptide I132-E152}

This peptide makes no contacts to the substrate analogues in the reported crystal structures, but forms part of the subunit interface. Trans-subunit conformational changes are known to occur with PNP, because the enzyme is capable of one-third the sites reactivity and inhibitor binding. The negative cooperativity between subunits requires trimeric conformational changes induced by interactions at a single subunit (Kline \& Schramm, 1992; Miles et al., 1998). In these $\mathrm{H} / \mathrm{D}$ exchange experiments, near-saturating concentrations of ligands are used, and subunit interface changes will be fully observed. The solvent exchange pattern for the intersubunit contact peptide is unlike the other peptides. Only 9 of the 17 amide hydrogens are exchanged after $3.5 \mathrm{~h}$ in the empty enzyme (Fig. 5). 


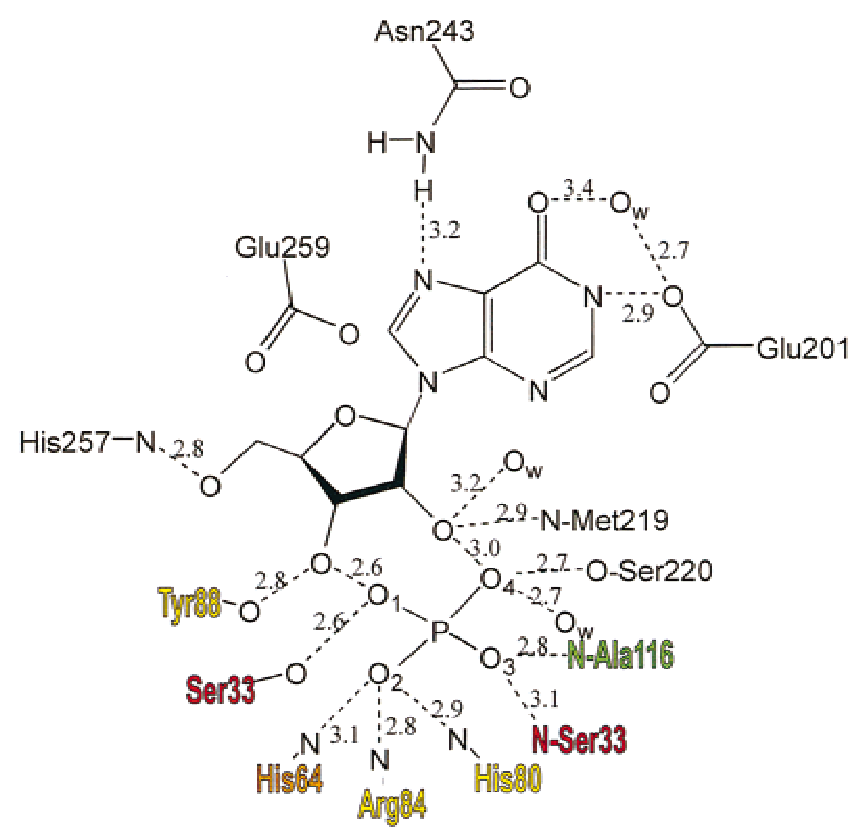

Fig. 9. Active site contacts of PNP with inosine and phosphate. Residues that hydrogen bond with the substrates are shown. Residues located in peptides identified by H/D exchange are color coded as in Figure 8.

Hypoxanthine, formycin $\mathrm{B}$, and immucillin- $\mathrm{H}$ all provide protection from exchange. Four additional amide hydrogens are buried in these complexes. Filling the catalytic sites causes this peptide to be nearly inaccessible to solvent, with only 5 of 17 amide protons exchanged after $2 \mathrm{~h}$. This peptide is the only one in which Michaelis and transition-state analogue inhibitors have similar effects. Conformational changes at this site in the subunit interface are, therefore, similar for both types of catalytic site ligands. The result indicates that the conformation changes at subunit interfaces are made by the Michaelis complex and that they are sustained through catalysis.

In all, binding of substrate analogues protects 10 amide protons from exchange at $2 \mathrm{~h}$ and 12 amide protons at $3.5 \mathrm{~h}$ with respect to controlled PNP involved. Among those 12 protected amide protons after exchange for $3.5 \mathrm{~h}$, four are located on three peptic peptides corresponding to residues 29-49, 50-70, and 81-98, which interact with the phosphate and ribosyl binding sites of PNP. Another four protons are located on peptic peptide 132-152, which is involved in the subunit interface near the catalytic site. The remaining four protons are on undefined peptides. Candidates include peptides that interact with the purine base, because none of these appear in the fragments identified by mass analysis. Purine base and nucleoside in the presence of sulfate (a phosphate analogue) cause equivalent protection. Thus, interactions between base, sulfate, and enzyme do not require ribosyl contacts to the enzyme to shield protein from solvent exchange.

Binding of transition state analogue immucillin-H inhibits 32 protons from solvent access at $2 \mathrm{~h}$ of exchange period and 29 protons at $3.5 \mathrm{~h}$. Among those 29 protected amide protons after exchange for $3.5 \mathrm{~h}, 11$ are located on four peptic peptides corresponding to residues $29-49,50-70,81-98$, and 112-124. Similarly to the Michaelis complex, another four protons are located on peptic peptide $132-152$. The remaining 14 protons are on undefined peptides.
Undefined peptides

The candidates for the 16 additional amide protons protected at $2 \mathrm{~h}$ by immucillin-H include the peptides that contain Glu201, Asn243, and His257, all of which make contacts to bound substrate analogues based on crystallographic studies (Koellner et al., 1997; Mao et al., 1998) (Fig. 9). A portion of the peptic digest of PNP remained insoluble under the conditions of the experiments. MALDI-TOF mass spectrometry analysis of these peptides solubilized with hexafluoroisopropanol showed a 4,260.8-Da fragment, corresponding to the sequence marked in Figure 4 from residues 240-277. The identification of this peptide sequence was also confirmed by Edman degradation. It did not prove possible to rapidly dissolve the insoluble peptides in an aprotic solvent, preventing further H/D exchange. Contacts between PNP and the transition-state analogue inhibitor provide additional binding energy. However, comparison between immucillin-H and inosine bound in the catalytic sites of PNP reveal similar peptide contacts, except that those between PNP and immucillin- $\mathrm{H}$ are most favorable (short hydrogen bonds) than to inosine (A. Federov, G. Kicska, E. Federov, W. Shi, P.C. Tyler, R.H. Furneaux, V.L. Schramm, \& S.C. Alma, unpubl. obs.). The similarity of the conformations suggests rate changes, rather than distinct conformations.

\section{Three-dimensional structure and kinetic mechanism}

The H/D exchange results for the five peptides are incorporated into the three-dimensional ribbon diagram derived from analysis of the crystal structure of the PNP trimer (Fig. 8). H/D exchange analysis by mass spectrometry identifies five peptides that experience changes in solvent accessibility as a consequence of binding of either product or analogues of substrate and transition state at the PNP active site. The position of these peptides in the X-ray crystal structure is within bonding distance of the substrate analogues, inosine, and inorganic sulfate. Peptide 132-152 occupies a position at the subunit interface that is likely to be protected during a conformational change associated with binding of substrate or product. Several amino acids in the catalytic site were not identified by the LC-MS analysis of peptic peptides. Of particular interest is Asn243, which interacts with the purine leaving group at the transition state (Erion et al., 1997). His257 is proposed to H-bond to the 5'-hydroxyl of inosine and has been suggested as the group causing a relatively large kinetic isotope effect as a result of this interaction (Kline \& Schramm, 1993). As noted above, during the 2-min peptic digestion, the PNP is fragmented partially, but a portion of the digest remains insoluble. The fragment containing Asn243 and His257 remains with the insoluble material.

The catalytic mechanism and structure of PNP have been examined by kinetic analysis, isotope effects, crystallography, and interaction with transition state analogue inhibitors. Based upon these experiments, the enzyme trimer functions by one-third-thesites reactivity in which each catalytic site functions sequentially. Under the incubation conditions used in the present experiments, product and inhibitor analogues were used at concentrations that would be bound to all sites of the PNP trimer. Hypoxanthine or immucillin-H binding occurs at only one of the three sites to provide full inhibition of the enzyme under defined conditions. However, this is a kinetically unstable state that leads to uniform site filling at sufficient inhibitor concentrations. Most of the experiments reported in this study were performed with an excess of substrates or inhibitors. However, the H/D exchange experiments 
for intact PNP trimer were also carried out at limiting immucillin-H, at one-third the concentration of the subunits (Fig. 2). Similar to the complex with saturating immucillin- $\mathrm{H}$, the deuterium incorporation decreases in the complex of one-third catalytic sites filled with immucillin-H, compared to free PNP or Michaelis complexes, and only a single molecular ion species was observed at each time point. If there is a heterogeneous structure with one tight subunit and two loose subunits present, a second envelope should be detected with a mass increase of around $27 \mathrm{Da}$. Filling the first, tightly bound site with immucillin-H limits access of all three polypeptides to solvent. The loss of flexibility prevents binding of inhibitor to sites two and three of the trimer, accounting for the negative cooperativity. The data obtained at short times of $\mathrm{H} / \mathrm{D}$ exchange were indistinguishable from those obtained with excess immucillin-H. However, at longer times of incubation, the two curves diverged somewhat. Immucillin-H protected 32 protons from exchange at all three sites filled and 27 positions from exchange at one-third the site inhibition at $2 \mathrm{~h}$ of exchange period. It may suggest that conformation of fully saturated complex is more stable than that of the complex of one-third the site inhibition. This result establishes a concerted change of subunit polypeptide conformation induced by a single catalytic site event.

\section{Conclusions}

H/D exchange in PNP is reduced by substrates, but more by a transition state analogue. Filling one site of the homotrimer with immucillin-H reduces $H / D$ exchange throughout the trimer, establishing a structural basis for the negative cooperativity of immucillin-H binding. The X-ray crystal structure for the Michaelis complexes indicates reduction of H/D exchange in peptides adjacent to the catalytic sites. Transition-state analogue interactions reduce the solvent accessibility of catalytic site peptides more than substrate or substrate analogues. A peptide involved in the subunit interface, but outside the catalytic site was equally influenced by ligands forming Michaelis or the transition state analogue complex.

\section{Materials and methods}

\section{Materials}

Purine nucleoside phosphorylase (PNP) from calf spleen in ammonium sulfate (Sigma Chemical Co., St. Louis, Missouri) was centrifuged and dissolved in $25 \mathrm{mM}$ HEPES buffer, $\mathrm{pH} 7.8$ to make a final concentration of $400 \mu \mathrm{M}$ subunits $(12.5 \mathrm{mg} / \mathrm{mL})$. Under these conditions, the concentration of sulfate ion is $\sim 200 \mathrm{mM}$ and is known to occupy the phosphate binding site on the enzyme (Mao et al., 1998). Pepsin was obtained from the Worthington Biochemical Co. (Freehold, New Jersey), and $\mathrm{D}_{2} \mathrm{O}$ (99.9 atom\% D) was from Isotec Inc. (Miamisburg, Ohio). Immucillin-H and ${ }^{14} \mathrm{C}$-immucillin-H were synthesized by a variation of published methods (Furneaux et al., 1997, 1999) and were characterized by NMR and high resolution mass spectrometry. All other chemicals and reagents were of the highest grade commercially available.

\section{Exchange studies}

$\mathrm{H} / \mathrm{D}$ exchange was initiated by dilution of $5 \mu \mathrm{L}$ of a $400 \mu \mathrm{M}$ solution of PNP in $25 \mathrm{mM}$ HEPES buffer, $\mathrm{pH} 7.8$ into $95 \mu \mathrm{L}$ of $25 \mathrm{mM}$ HEPES buffer in 99 atom\% excess $\mathrm{D}_{2} \mathrm{O}, \mathrm{pD} 7.8$ to give a final enzyme concentration of $20 \mu \mathrm{M}$. The complex of PNP with hypoxanthine was formed by $110 \mu \mathrm{M}$ hypoxanthine in the deuterated buffer. That with formycin B was formed by $2 \mathrm{mM}$ formycin B in the deuteration buffer. The complex of PNP-immucillin- $\mathrm{H}$ forms in a time-dependent fashion (Miles et al., 1998). It was formed by incubating $400 \mu \mathrm{M}$ PNP and $800 \mu \mathrm{M}$ immucillin- $\mathrm{H}$ at $4{ }^{\circ} \mathrm{C}$ for greater than $1 \mathrm{~h}$ prior to dilution into deuteration buffer. Some experiments were also performed at limiting concentrations of inhibitor to onethird the total concentration of the PNP subunits. Solutions were maintained at room temperature for $\mathrm{H} / \mathrm{D}$ exchange and allowed to exchange for the desired times. At appropriate intervals, aliquots of the PNP solution were adjusted to $\mathrm{pH} 2.2$ by the addition of an equal volume of $0.5 \mathrm{M}$ ammonium phosphate, $\mathrm{pH} 2.2$, and immediately cooled to $0{ }^{\circ} \mathrm{C}$. Samples were analyzed by LC-MS analysis or peptic digestion and subsequent LC-MS analysis to determine the extent of deuterium incorporation into PNP or the peptic peptides. Exchanged solutions of PNP were simultaneously quenched and digested by adding an equal volume of $0.5 \mathrm{M}$ ammonium phosphate buffer, $\mathrm{pH} 2.2$, containing $10 \mu \mathrm{M}$ pepsin, followed by incubation for $2 \mathrm{~min}$ at $0{ }^{\circ} \mathrm{C}$ to effect proteolysis.

\section{LC-MS analysis of the deuterated PNP and its peptic peptides}

The extent of deuterium incorporation into PNP and specific peptides of PNP was determined by HPLC coupled with mass spectrometry (LC-MS) analysis of deuterated PNP and its peptic peptides. An HP 1100 HPLC equipped with the degasser and binary pump was used to on-line degas the solvents and pump the solvents to generate acetonitrile gradients. An Acurate IC-100-VAR splitter with $1.0 \mathrm{~mm}$ I.D. column calibrator from the LC Packings (San Francisco, California) was placed between the mixer of HPLC and the injector to minimize the delay time of the mixer. The flow at $50 \mu \mathrm{L} /$ min went to the LC injector following the splitter. The splitter calibration loop, injector, and column were all immersed in an ice bath $\left(0{ }^{\circ} \mathrm{C}\right)$ to minimize back exchange of deuterium for hydrogen with HPLC solvents. The exchanged and quenched protein solution $(20 \mu \mathrm{L})$ or peptic digests $(50 \mu \mathrm{L})$ were loaded onto Vydac $1.0 \times$ $50 \mathrm{~mm} \mathrm{C}_{4}$ or $1.0 \times 50 \mathrm{~mm} \mathrm{C}_{8}$ columns (The Separations Group, Hesperia, California), respectively. Solvent A is $5 \%$ acetonitrile in water containing $0.05 \%$ trifluoroacetic acid, and solvent $\mathrm{B}$ is $95 \%$ acetonitrile containing $4.95 \% \mathrm{H}_{2} \mathrm{O}$ and $0.05 \%$ trifluoroacetic acid. After desalting for $2 \mathrm{~min}(0 \% \mathrm{~B})$, the protein eluted at $\sim 11 \mathrm{~min}$ with a 2 min $0-100 \%$ B gradient. The peptic peptides eluted between 11 and 13 min with a $10 \mathrm{~min} 5-60 \%$ B gradient after desalting for $2 \mathrm{~min}$ at $5 \% \mathrm{~B}$. The column effluent $(50 \mu \mathrm{L} / \mathrm{min})$ was delivered directly to the mass spectrometer without flow splitting. The mass resolution of the spectrometer was tuned to give a constant peak width of $1 \mathrm{Da}$ (full-width at half-maximum) across the mass range of interest. Full scan mass spectra were acquired using a step size of $0.1 \mathrm{Da}$ with a scan time of $5.64 \mathrm{~s}$ across the mass range of $950-1,225$ for the protein sample, and with a scan time of $4.31 \mathrm{~s}$ across the mass range of $520-1,300$ for the peptic digests.

The number of deuterium exchanged into peptide amide positions (D) in PNP or PNP-derived peptic peptides was determined from the mass difference between nondeuterated and deuterated samples after correction for the loss of deuterium during LC-MS analysis or peptic digestion and subsequent LC-MS analysis (Zhang \& Smith, 1993). Equation 1 is used for the calculation of the number of deuterium incorporated,

$$
D=\frac{m_{t}-m_{0}}{m_{100}-m_{0}} * N
$$


where $m_{t}$ is the protein (peptide) mass at time $t, m_{0}$ is the protein mass at time zero, $m_{100}$ is the protein mass at infinite time (completely exchanged), and $N$ is the total number of exchangeable amide protons. The masses used in Equation 1 are experimental masses measured by on line LC-MS. Completely deuterated PNP was obtained by incubating PNP in $2 \mathrm{M}$ urea- $\mathrm{d}_{4}$ at $55^{\circ} \mathrm{C}$ for $1 \frac{1}{2} \mathrm{~h}$. The fully exchanged PNP was digested with pepsin, and D for $\mathrm{H}$ back exchange of peptic peptides in this study was determined from the theoretical mass for the completely amide-deuterated fragment and the experimental mass determined after LC-MS analysis. For the peptides described here, the extent of back exchange varied between 32 and 58\% during peptic digestion and LC-MS analysis. This value is consistent with $40-50 \%$ of back-exchange reported by other authors (Johnson \& Walsh, 1994; Johnson, 1996). Kinetic analysis of peptides exchanged was performed as described by Zhang et al. (1997).

\section{Mass spectrometry and tandem mass spectrometry}

All mass spectra were acquired using an API-III triple quadrupole mass spectrometer equipped with an atmospheric pressure ionization (API) source and a pneumatically assisted electrospray interface (PE-SCIEX, Thornhill, ON, Canada). High purity nitrogen was used as the nebulizing gas to prevent back exchange of deuterium by ambient air during the ionspray process. A Macintosh Quadra 950 was used for instrument control, data acquisition, and processing. Tandem mass spectrometry was performed to identify peptic peptides. Fragment ion (tandem) mass spectra were obtained using collision-induced dissociation of precursor ions selected by their $\mathrm{m} / \mathrm{z}$ value in the first quadrupole. Collisional activation was accomplished by introducing argon into the second (rf-only) quadrupole, and the resulting fragment ions were analyzed in the third quadrupole. Optimum collision conditions varied with the precursor peptides, with target gas thickness ranging from $1.5-4 \times 10^{14}$ molecules $/ \mathrm{cm}^{2}$ and accelerating potential differences into the collision cell in the range of $20-80 \mathrm{eV}$.

\section{Equilibrium binding of ${ }^{14} \mathrm{C}$-immucillin- $\mathrm{H}$ to $\mathrm{PNP}$}

Samples buffered with $10 \mathrm{mM}$ potassium phosphate $\mathrm{pH} 7.5$ contained $100 \mu \mathrm{L}$ of $0.05-10 \mu \mathrm{M}$ PNP, $0.05-20 \mu \mathrm{M}\left[{ }^{14} \mathrm{C}\right]$-immucillin$\mathrm{H}$ mixed with cold immucillin-H. Samples were loaded onto centrifugal filters, Microcon 10,000 MWCO (Millipore, Massachusetts). Binding was determined by comparing ${ }^{14} \mathrm{C}$ content of sample before and after filtration (Schramm, 1976). Label (1 nM ${ }^{32} \mathrm{P}$-phosphate) was used to establish the sample dilution caused by the filter.

\section{Acknowledgments}

This work was supported by NIH Research Grant GM41916 (VLS), shared instrumentation grants from the NIH (RR-09113, R.H.A.) and NSF (BIR9318193, R.H.A.), and a grant from the Mathers Foundation (V.L.S.).

\section{References}

Biemann K. 1988. Contributions of mass spectrometry to peptide and protein structure. Biomed Environ Mass Spectrom 16:99-111.

Erion MD, Stoeckler JD, Guida WC, Walter RL, Ealick SE. 1997. Purine nucleoside phosphorylase. 2. Catalytic mechanism. Biochemistry 36:1173511748 .
Furneaux RH, Limberg G, Tyler PC, Schramm VL. 1997. Synthesis of transition state inhibitors for N-riboside hydrolases and transferases. Tetrahedron 53:2915-2930

Furneaux RH, Schramm VL, Tyler PC. 1999. Transition state analogue inhibitors of protozoan nucleoside hydrolases. Bioorg Med Chem 7:2599-2606.

Hershfield MS, Mitchell BS. 1995. Immunodeficiency diseases caused by adenosine deaminase deficiency and purine nucleoside phosphorylase deficiency. In: Scriber CR, Beaudet AL, Sly WS, Valle D, eds. The metabolic basis of inherited disease, 7th ed. New York: McGraw-Hill. pp 1725-1768.

Johnson RS. 1996. Mass spectrometric measurement of changes in protein hydrogen exchange rates that result from point mutation. J Am Soc Mass Spectrom 7:515-521.

Johnson RS, Walsh KA. 1994. Mass spectrometric measurement of protein amide hydrogen exchange rates of apo- and holo-myoglobin. Protein Sci 3:2411-2418.

Katta V, Chait BT. 1993. Hydrogen/deuterium exchange electrospray ionization mass spectrometry: A method for probing protein conformational changes in solution. J Am Chem Soc 115:6317-6321.

Kline PC, Schramm VL. 1992. Purine nucleoside phosphorylase. Inosine hydrolysis, tight binding of the hypoxanthine intermediate, and third-the-sites reactivity. Biochemistry 31:5964-5973.

Kline PC, Schramm VL. 1993. Purine nucleoside phosphorylase. Catalytic mechanism and transition state analysis of the arsenolysis reaction. Biochemistry 32:13212-13219.

Koellner G, Luic M, Shugar D, Saenger W, Bzowska A. 1997. Crystal structure of calf spleen purine nucleoside phosphorylase in a complex with hypoxanthine at 2.15 A resolution. J Mol Biol 265:202-216.

Mandell JG, Falick AM, Komives EA. 1998a. Measurement of amide hydrogen exchange by MALDI-TOF mass spectrometry. Anal Chem 70:3987-3995.

Mandell JG, Falick AM, Komives EA. 1998b. Identification of protein-protein interfaces by decreased amide proton solvent accessibility. Proc Natl Acad Sci USA 95:14705-14710.

Mao C, Cook WJ, Zhou M, Federov AA, Almo SC, Ealick SE. 1998. Calf spleen purine nucleoside phosphorylase complexed with substrates and substrate analogues. Biochemistry 37:7135-7146.

Miles RW, Tyler PC, Furneaux RH, Bagdassarian CK, Schramm VL. 1998. One-third-the-sites transition state inhibitors for purine nucleoside phosphorylase. Biochemistry 37:8615-8621.

Morris PE Jr, Montgomery JA. 1998. Inhibitors of the enzyme purine nucleoside phosphorylase. Exp Opin Ther Patents 8:283-299.

Morrison JF, Walsh CT. 1988. The behavior and significance of slow-binding enzyme inhibitors. Adv Enzymol Relat Areas Mol Biol 61:201-301.

Schramm VL. 1976. Comparison of initial velocity and binding data for allosteric adenosine monophosphate nucleosidase. J Biol Chem 10:3417-3424.

Smith DL, Deng Y, Zhang Z. 1997. Probing the non-covalent structure of proteins by amide hydrogen exchange and mass spectrometry. J Mass Spectrom 32:135-146.

Wang F, Blanchard JS, Tang XJ. 1997. Amide hydrogen exchange/electrospray ionization mass spectrometry studies of substrate and inhibitor binding and conformational changes of E. coli dihydropicolinate reductase. Biochemistry 36:3755-3759.

Wang F, Li W, Emmett MR, Hendrickson CL, Marshall AG, Zhang YL, Wu L, Zhang ZY. 1998a. Conformation and dynamic changes of Yersinia protein tyrosine phosphatase induced by ligand binding and active site mutation and revealed by $\mathrm{H} / \mathrm{D}$ exchange and electrospray ionization Fourier transform ion cyclotron resonance mass spectrometry. Biochemistry 37:15289-15299.

Wang F, Li W, Emmett MR, Marshall AG, Corson D, Sykes BD. 1999. Fourier transform ion cyclotron resonance mass spectrometric detection of small $\mathrm{Ca}^{2+}$-induced conformation changes in the regulatory domain of human cardiac troponin C. J Am Soc Mass Spectrom 10:703-710.

Wang F, Scapin G, Blanchard JS, Angeletti RH. 1998b. Substrate binding and conformational changes of Clostridium glutamicum diaminopimelate dehydrogenase revealed by hydrogen/deuterium exchange and electrospray mass spectrometry. Protein Sci 7:293-299.

Woodward C. 1999. Advances in protein hydrogen exchange by mass spectrometry. J Am Soc Mass Spectrom 10:672-674.

Zhang Z, Li W, Logan WM, Li M, Marshall AG. 1997. FK506-binding protein amide hydrogen exchange rates from mass spectrometry match and extend those from NMR. Protein Sci 6:2203-2217.

Zhang Z, Post CB, Smith DL. 1996. Amide hydrogen exchange determined by mass spectrometry: Application to rabbit muscle aldolase. Biochemistry 35:779-791.

Zhang Z, Smith DL. 1993. Determination of amide hydrogen exchange by mass spectrometry: A new tool for protein structure elucidation. Protein Sci 2:522531. 